\section{O processo de implantação dos comitês de investigação do óbito infantil no Estado de São Paulo}

\section{The installation of Child Mortality Investigation Committees in the State of São Paulo}

Sonia Isoyama Venâncio 1

Rui de Paiva 2

1,2 Instituto de Saúde. Secretaria de Estado da Saúde de São Paulo.
Rua Santo Antonio, 590. Bela Vista. São Paulo, SP, Brasil. CEP: 01314-000. E-mail: soniav@isaude.sp.gov.br

\begin{abstract}
Objectives:to evaluate the installation of Child Mortality Investigation Committees (CMIOs) in the State of São Paulo (SP).

Methods: the study was carried out in two stages: a) a survey to establish whether committees exist in all Regional Health Directorates (RHD) and municipalities in the State; b) focus groups with members of the regional and municipal committees to identify any difficulties that have arisen.

Results: $87 \%$ of RHDs and $53 \%$ of municipalities were found to have CMIOs, most of which were set up between 2003 and 2005. The main difficulties mentioned during the focus groups were as follows: lack of physical and administrative structure; activities conducted on a one-off basis on the initiative of individuals or small groups; lack of support for higher levels in the hierarchy; actions not considered during planning; lack of coordination, support and articulation of infant mortality reduction strategies at State level.

Conclusions: a high degree of compliance with CMIO installation was found at regional level and an average degree at municipal level. The regional and municipal committees both lack infrastructure and experience technical, operational and political difficulties that may compromise their role in reducing child mortality.
\end{abstract}

Key words Infant mortality, Committees for Research on Child Death

\section{Resumo}

Objetivos: avaliar a implantação dos Comitês de Investigação do Óbito Infantil (CIOI) no Estado de São Paulo (SP).

Métodos: a pesquisa foi realizada em duas etapas: a) levantamento sobre a existência dos comitês em todas as Diretorias Regionais de Saúde (DIR) e municipios do Estado; b) grupos focais com membros de comitês regionais e municipais para identificação de dificuldades no processo de trabalho dos mesmos.

Resultados: em $87 \%$ das DIR e 53\% dos municipios existiam CIOI que foram implantados majoritariamente nos anos de 2003 a 2005. As principais dificuldades reveladas nos grupos focais foram: falta de estrutura material e administrativa; atividades conduzidas por iniciativas isoladas de pessoas ou pequenos grupos; falta de respaldo dos niveis superiores de hierarquia; ações não contempladas nas instâncias de planejamento; ausência de coordenação, apoio e articulação de estratégias de redução da mortalidade infantil em nivel estadual.

Conclusões: verificou-se uma grande adesão à implantação dos CIOI em SP no nivel regional, sendo intermediária no nível municipal. Os comitês regionais e municipais apresentaram infraestrutura insuficiente e problemas técnico-operacionais e políticos que podem comprometer seu papel na redução da mortalidade infantil.

Palavras-chave Mortalidade infantil, Comitês de Investigação do Óbito Infantil 


\section{Introdução}

De modo geral, a mortalidade infantil (MI) está em queda no mundo, o mesmo ocorrendo no Brasil. ${ }^{1}$ Seu movimento declinante vem revelando nuances sobre o acesso de diferentes camadas da população aos recursos de assistência à saúde. 2,3

Signatário dos Objetivos de Desenvolvimento do Milênio em 2000, o país impôs-se, entre outras metas, a de "reduzir em dois terços, entre 1990 e 2015, a mortalidade de crianças menores de cinco anos (MM5)". Dados de 2005 revelam queda de $46,4 \%$ da MM5, com destaque para o nordeste $(55,4 \%)$. A taxa de mortalidade infantil (TMI) caiu em 37,2\% entre 1996 e 2005 (de 33,7/1000 a 21,1/1000), embora persistam importantes desequilíbrios regionais. Além disso, a TMI pode ser considerada elevada quando comparada a de países desenvolvidos e alguns sul-americanos, como Chile e Argentina (16/1000 e 8/1000, respectivamente, em 2008). 4,5

No Estado de São Paulo (SP), a queda da TMI foi acentuada, de 30,1\% de 1998 a $2007(18,7 / 1000$ e 13,07/1000, respectivamente) e tem sido sistemática desde meados da década de 1970. Por suas características populacionais e econômicas, a comparação com outras localidades não é positiva. 6,7 Mantido o ritmo atual de queda da TMI $(-2,8 \%$ no quinquênio 2003-2007), SP, cujo índice de desenvolvimento humano (IDH) era 0, 833 em 2007, demoraria mais de vinte anos para atingir a marca de 7/1000, que o Chile atingiu em 2008, com IDH semelhante $(0,878)$.

A investigação das mortes infantis vem sendo recomendada de forma crescente em vários documentos legais no âmbito do Sistema Único de Saúde (SUS) desde 2004, como estratégia que pode contribuir para a adoção de medidas de prevenção de óbitos evitáveis pelos serviços de saúde.4,8-11

Desde 2002 foram divulgados pelo Estado de SP um manual prático dos comitês de mortalidade infantil e fichas de investigação do óbito, elaborados pela área técnica de saúde da criança da Secretaria Estadual de Saúde (SES-SP), material de apoio inspirado em experiências anteriores que visavam auxiliar a implantação e funcionamento dos grupos de investigação estadual.12-14 A ausência de um Comitê Estadual em SP, que foi criado somente em setembro de 200615 e começou suas atividades em 2007, dificultou o acompanhamento de sua implantação nas regiões e municípios do Estado.

Nesse contexto, com objetivo de analisar o processo de implantação dos Comitês de Investigação do Óbito Infantil (CIOI) nas 24 regiões de saúde do Estado de SP nos anos de 2005-2006 e identificar as dificuldades ocorridas para a consolidação da estratégia no SUS-SP, desenvolveu-se o presente estudo.

\section{Métodos}

Pesquisa realizada em duas etapas: a) primeira etapa: estudo descritivo de corte transversal, no qual foram coletadas informações sobre a implantação dos CIOI na totalidade das regiões e municípios do Estado de São Paulo. Nessa etapa foi utilizado um questionário semiestruturado dirigido às 24 Diretorias Regionais de Saúde (DIR) e aos 645 municípios, cabendo às DIR a responsabilização pelo envio e recolhimento dos mesmos. Os questionários continham perguntas sobre o período de início das atividades dos comitês; mecanismos de investigação do óbito infantil; instrumentos de análise utilizados; composição dos comitês; ações desencadeadas pelos comitês municipais visando à redução da mortalidade infantil e as principais dificuldades encontradas nesse processo. Os questionários foram digitados no programa Epi Info 2002 para posterior análise descritiva. Considerou-se que o Comitê estava implantado quando por ocasião da pesquisa se declarou sua existência e o desenvolvimento de atividades de investigação dos óbitos, independente da formalização dos mesmos e de implantação intermediária se apesar da existência do mesmo as atividades de investigação eram incipientes; b) segunda etapa: realizou-se o aprofundamento da percepção dos atores envolvidos sobre o processo de investigação dos óbitos infantis, através de estudos de caso em três regiões de saúde do Estado de SP. As regiões foram selecionadas buscando-se abranger diferentes contextos, obedecendo aos critérios de situação da mortalidade infantil (taxas acima e abaixo da média estadual) e facilidade de acesso para a realização dos grupos: Mogi das Cruzes (Região Metropolitana da Grande São Paulo); Santos (Região Metropolitana da Baixada Santista), ambas com TMI acima da média estadual e Barretos (interior do Estado de SP), eleita pelo comportamento de sua TMI, que em uma década equiparou-se às melhores regiões do Estado.

A técnica qualitativa utilizada para a coleta dos dados foi a de entrevistas de grupo por meio de grupos focais. ${ }^{16}$ Realizou-se um grupo focal em cada DIR, com membros dos comitês regional e municipais, totalizando entre 12 a 15 participantes. Um roteiro de discussão foi previamente elaborado, elegendo como questões norteadoras: o papel dos comitês, sob a ótica dos participantes e os fatores que dificultavam o trabalho. Os grupos foram 
conduzidos por um moderador e um observador; as falas foram gravadas e posteriormente transcritas. ${ }^{17}$

Os dados foram colhidos no segundo semestre de 2005 e primeiro semestre de 2006. A DIR I, composta pela capital, não aceitou participar do estudo. A pesquisa foi aprovada pelo Comitê de Ética do Instituto de Saúde - SES-SP.

\section{Resultados}

No nível regional, verificou-se a existência de CIOI em $87 \%$ das DIR (Tabela 1), sendo que em duas delas as atividades eram ainda incipientes. O primeiro comitê surgiu em 1999 e 14 foram implantados entre anos de 2003 a 2005. Os maiores responsáveis pela implantação, isoladamente, foram os diretores de DIR (55\%) ou diretores de DIR e áreas técnicas de saúde da criança (65\%). A participação da vigilância epidemiológica na responsabilidade pela condução dos trabalhos foi baixa (32\%), deixando à área técnica (AT) encarregada de $64 \%$ da investigação dos óbitos.

A composição dos CIOI foi variada, com forte predominância das representações de profissionais da saúde (médicos e enfermeiros), acentuando uma característica técnica e de comitês grandes, com mais de 15 membros (48\% das Regionais); em 45\% das DIR as reuniões eram realizadas em período bimensal; $57 \%$ investigavam todos os casos de óbitos e menos de $20 \%$ utilizavam algum critério de seleção de casos (evento sentinela, peso ao nascer, causa mortis, idade gestacional). Quanto ao material padronizado para a investigação e análise dos casos, $55 \%$ utilizavam formulários da área técnica da SESSP; $40 \%$ próprios da DIR e $25 \%$ do Ministério da Saúde. Metade dos grupos fazia entrevistas com

\section{Tabela 1}

Implantação dos comitês de investigação do óbito infantil nas regionais de saúde do Estado de São Paulo. São Paulo, 2006.

\begin{tabular}{|c|c|c|c|}
\hline \multirow[t]{2}{*}{ Diretorias Regionais de Saúde } & \multicolumn{3}{|c|}{ Implantação dos comitês } \\
\hline & Implantado & Parcialmente implantado & Não Implantado \\
\hline DIR I - São Paulo & $x$ & & \\
\hline DIR II - Mogi das Cruzes & & $x$ & \\
\hline DIR IIII - Santo André & & $x$ & \\
\hline DIR IV - Franco da Rocha & $x$ & & \\
\hline DIR V - Osasco & $x$ & & \\
\hline DIR VI - Araçatuba & $x$ & & \\
\hline DIR VII - Araraquara & $x$ & & \\
\hline DIR VII - Assis & $x$ & & \\
\hline DIR IX -Barretos & $x$ & & \\
\hline DIR X - Bauru & $x$ & & \\
\hline DIR XI - Botucatu & $\mathrm{x}$ & & \\
\hline DIR XII-Campinas & & & $x$ \\
\hline DIR XIII - Franca & $x$ & & \\
\hline DIR XIV - Marília & $x$ & & \\
\hline DIR XV - Piracicaba & $x$ & & \\
\hline DIR XVI - Presidente Prudente & $x$ & & \\
\hline DIR XVII - Registro & $x$ & & \\
\hline DIR XVII - Ribeirão Preto & $x$ & & \\
\hline DIR XIX - Baixada Santista & $x$ & & \\
\hline DIR XX - São João da Boa vista & $x$ & & \\
\hline DIR XXI - São José dos Campos & $x$ & & \\
\hline DIR XXII - São José do Rio Preto & $x$ & & \\
\hline DIR XXIII - Sorocaba & $x$ & & \\
\hline DIR XXIV - Taubaté & $x$ & & \\
\hline
\end{tabular}

DIR=Diretorias Regionais de Saúde. 
profissionais que haviam atuado no caso estudado, mesma proporção dos que faziam visita domiciliar; em $80 \%$ das regionais era realizada uma síntese de cada caso para focalizar as possibilidades de intervenção, havendo análise prévia do comportamento da MI em $85 \%$ delas e relatos de alguma modificação na assistência decorrentes da ação dos CIOI em $63 \%$.

No plano municipal, havia CIOI em $53 \%$ dos municípios (Tabela 2), sendo o primeiro implantado em 1992 e 153 entre os anos de 2003 a 2005. Em $40 \%$ deles o secretário de saúde foi o maior responsável pela implantação e em $63 \%$ a vigilância epidemiológica respondia pela investigação dos óbitos. A composição nos municípios também mostra comitês grandes, com mais de 15 membros em $76 \%$ deles e reuniões em período bimensal em $66 \% ; 90 \%$ dos municípios investigavam todos os casos; $30 \%$ usavam formulário da DIR; $23,4 \%$ do
MS; $13,3 \%$ da área técnica da SES-SP e apenas $10,4 \%$ formulário próprio; $61 \%$ faziam entrevistas com profissionais que haviam atuado no caso e $91 \%$ faziam visita domiciliar e houve referência ao uso de síntese do caso em $73 \%$ dos municípios; $61 \%$ dos comitês municipais fizeram uma análise prévia do comportamento da MI e apenas 37\% relataram alguma modificação na assistência decorrente das ações dos CIOI.

As falas dos participantes dos grupos focais revelaram importantes dificuldades para o funcionamento e realização de ações dos comitês. Foi desvelada a insatisfação pela insuficiência de estrutura organização ou recursos materiais, seja no nível regional ou nos pequenos municípios, que são maioria em SP. A deficiência de recursos é citada com muita frequência como um obstáculo a uma ação mais produtiva:

Tabela 2

Percentual de municípios com implantação de comitês na região de saúde do Estado de São Paulo.

\begin{tabular}{|c|c|c|c|}
\hline Diretorias Regionais de Saúde & $\begin{array}{c}\text { Total de } \\
\text { municípios }\end{array}$ & $\begin{array}{c}\text { Número de } \\
\text { municípios com } \\
\text { comitê implantado }\end{array}$ & $\begin{array}{l}\text { Percentual de } \\
\text { municípios com } \\
\text { comitê implantado }\end{array}$ \\
\hline DIR I - São Paulo & 1 & 1 & 100,0 \\
\hline DIR II - Santo André & 7 & 6 & 85,7 \\
\hline DIR III - Mogi das Cruzes & 11 & 7 & 63,6 \\
\hline DIR IV - Franco da Rocha & 5 & 2 & 40,0 \\
\hline DIR IX - Barretos & 19 & 17 & 89,5 \\
\hline DIR V - Osasco & 15 & 12 & 80,0 \\
\hline DIR VI - Araçatuba & 40 & 32 & 80,0 \\
\hline DIR VII - Araraquara & 25 & 11 & 44,0 \\
\hline DIR VIII - Assis & 25 & 4 & 16,0 \\
\hline DIR X - Bauru & 38 & 21 & 55,3 \\
\hline DIR XI - Botucatu & 30 & 2 & 6,7 \\
\hline DIR XII - Campinas & 42 & 19 & 45,2 \\
\hline DIR XIII - Franca & 22 & 13 & 59,1 \\
\hline DIR XIV - Marília & 37 & 9 & 24,3 \\
\hline DIR XIX - Santos & 9 & 8 & 88,9 \\
\hline DIR XV - Piracicaba & 26 & 7 & 26,9 \\
\hline DIR XVI - Presidente Prudente & 45 & 0 & - \\
\hline DIR XVII - Registro & 15 & 4 & 26,7 \\
\hline DIR XVIII - Ribeirão Preto & 25 & 8 & 32,0 \\
\hline DIR XX - São João da Boa Vista & 20 & 6 & 30,0 \\
\hline DIR XXI - São José dos Campos & 12 & 6 & 50,0 \\
\hline DIR XXII - São José do Rio Preto & 101 & 94 & 93,1 \\
\hline DIR XXIII - Sorocaba & 49 & 48 & 98,0 \\
\hline DIR XXIV - Taubaté & 26 & 6 & 23,1 \\
\hline Total & 645 & 343 & 53,2 \\
\hline
\end{tabular}

DIR=Diretorias Regionais de Saúde. 
"É uma coisa muito pesada... Eu não sei a DIR de vocês, mas a gente não tem retaguarda administrativa... E é o técnico que assume tudo... Mas é difícil, não tem computador e Internet para mandar correspondência..."

O velho e recorrente problema dos trabalhadores em sistemas públicos com seus baixos níveis de responsabilidade/autoridade também surgiu. As ações são desencadeadas por pessoas que chamam para si a responsabilidade, mas nem sempre têm poder necessário para desempenhar a tarefa ou determinar a participação de outros servidores:

[...] conversamos com os municípios sempre baseados no manual de investigação de óbitos infantis, e os municípios... os técnicos, a gente fala município, mas não é bem município, são aqueles técnicos que estão envolvidos com as ações e eles acabam chamando para si aquela ação..

O "nível central", como se referem os servidores aos níveis hierárquicos superiores nas secretarias municipais e instâncias regionais, também teve sua quota de menção, no tocante ao envolvimento com o trabalho dos comitês. De novo surge a questão de responsabilidade sobre o desencadeamento de ações a partir das conclusões do CIOI:

[...] faz um comitê no papel. Tem as pessoas que investigam o óbito infantil, investigam a morte materna, mas nestes comitês para discutir o que está acontecendo existe sempre muito boa vontade, mas nunca resolve nada... as ações desencadeadas tiveram algum impacto? Não, porque não houve ação.... Não existe nenhum envolvimento do nível central...

Esse distanciamento dos níveis hierárquicos superiores das necessidades e/ou ações indicadas pelos CIOI, o funcionamento com carência global de recursos, apoiado muitas vezes no voluntarismo de servidores dedicados, caracterizaram uma falta de institucionalidade dos comitês. Isso se traduziu, ainda, no plano político-administrativo, em falas que enfatizavam a importância da atuação de autoridades como pólos políticos de poder. Esse tom, porém, foi quase sempre de lamento, queixa, crítica velada ou franca:

[...] investiga um pouquinho e não tem com quem discutir... A gente faz relatório e manda para o Secretário e ele não se manifesta, põe na gaveta e acho que não sabe qual é o nosso objetivo e porque foi criado. A gente sabe o que está acontecendo e não tem como melhorar, e se pensa: o que estamos fazendo ali afinal?
Evidenciou-se, finalmente, o reconhecimento da institucionalidade como forma de resolução dessa dicotomia (necessidades técnicas versus ações políticas), na forma de gestores atuantes, com políticas definidas e ações claras, para enfrentar um problema que, embora complexo, é dado como suficientemente compreendido:

[...] a primeira coisa que até é bem claro e evidente, é que quando se tem um gestor envolvido e preocupado que as coisas aconteçam no momento certo e dando condições para que todo o processo que se desencadeia [...] o município vai bem.

\section{Discussão}

Verificou-se grande adesão do nível regional e adesão intermediária do nível municipal à implantação dos CIOI no Estado de SP no período analisado. Os dados apresentados mostram, além da implantação parcial dos CIOI, dificuldades internas com repercussão em suas ações e possíveis resultados. Parece não haver reconhecimento de que os comitês grandes, com reuniões esparsas, sem um critério de seleção de óbitos a serem estudados, trabalhando com manuais e fichas não padronizados, têm suas possibilidades de efetividade reduzidas. O achado de que $39 \%$ dos municípios que implantaram o CIOI sequer haviam estudado o comportamento histórico da TMI em seu território parece revelador de limitações intrínsecas importantes.

Segundo o MS, a proporção de óbitos infantis e fetais investigados no país ainda é baixa. Porém, as primeiras iniciativas de implantação dos comitês a partir de meados da década de 1990 e a elaboração do manual dos comitês de prevenção do óbito infantil e fetal em 2004, como referência para sua estruturação, vêm tornando essa estratégia uma realidade. Pesquisa com características similares a esta, realizada em 2007 pelo MS em parceria com centros colaboradores de saúde da criança e centros universitários, nos municípios com população de 80.000 habitantes ou mais, mostrou que cerca de $50 \%$ destes municípios já tinham comitês, com uma proporção maior nas Regiões Sul e Sudeste. ${ }^{8}$ Corroborando este estudo, também foi evidenciado a necessidade de maior apoio técnico e operacional das secretarias de estado, MS e gestores municipais para qualificar o trabalho dos grupos de investigação, o que sugere a existência de fragilidades neste processo.

Para buscar resultados concretos, os comitês precisariam de implantação ampla, papel conceitualmente claro, infraestrutura adequada, além de ser, efetivamente, um pólo técnico/gerencial cujas ações 
ou recomendações fluíssem com total respaldo das autoridades. Isso daria o sentido de esmiuçar o processo assistencial em cada óbito, obtendo elementos para a avaliação da qualidade em seus componentes de estrutura, processo e resultado, além de propor as alterações necessárias. 18

Falta de estrutura material e administrativa (salas, computadores, softwares, internet, pessoas), atividades conduzidas por iniciativas isoladas de pessoas ou pequenos grupos, sem respaldo dos níveis superiores de hierarquia, ações não contempladas nas instâncias de planejamento, desmotivação e baixa responsabilização dos protagonistas são os principais fatores que caracterizam uma não institucionalidade dos CIOI, retirados das falas analisadas. Tal condição dessa estratégia de enfrentamento da mortalidade infantil mostra como podem ser frágeis as ações concretas institucionais, ainda que a TMI seja um indicador sempre presente nos documentos do setor saúde.

Considerando que a principal função dos comitês seria "mobilizar parceiros e propor medidas para a redução da mortalidade"8 e que, através da

\section{Referências}

1. Black R E, Morris SS, Bryce J. Where and why are 10 million children dying every year? Lancet. 2003; 361: 2226-34. Disponível em http://www.thelancet.com/journal/ vol361/iss9376/contents

2. The Bellagio Study Group on Child Survival. Knowledge into action for child survival. Lancet. 2003; 362: 323-7.

3. Mello JMHP, Gotlieb SLD. As condições de saúde no Brasil. Ministério da Saúde. Rio de Janeiro: Fiocruz; 2000. p. 164-5.

4. Objetivos de desenvolvimento do milênio. Relatório Nacional de Acompanhamento. Setembro de 2004. IPEA/IBGE/Presidência da República.

5. Objetivos de desenvolvimento do milênio. 3o Relatório Nacional de Acompanhamento. Setembro de 2007. IPEA/IBGE/Presidência da República.

6. Paiva R. Evolução da mortalidade infantil no município de São Paulo nas últimas três décadas [dissertação]. São Paulo: Faculdade de Saúde Pública da Universidade de São Paulo; 2002

7. UNICEF. Childinfo: monitoring situation of children and women (last update November 2009). [Acesso em $10 \mathrm{dez}$ 2009]. Disponível em http://www.childinfo.org/mortality_ imrcountrydata.php.

8. Brasil. Ministério da Saúde. Manual de vigilância do óbito infantil e fetal e do comitê de prevenção do óbito infantil e fetal. (Série A. Normas e Manuais Técnicos). Brasília, DF; 2009

9. Brasil. Ministério da Saúde. Pacto nacional pela redução da mortalidade materna e neonatal. Brasília, DF; 2004.

10. Brasil. Ministério da Saúde. Manual dos comitês de morte materna. Brasília, DF; 2002. vigilância dos óbitos é possível identificar a precariedade do acesso e da qualidade da atenção prestada às crianças, ${ }^{19}$ sua implantação deveria ter, como resultado, a redução da mortalidade infantil. Porém, faltam estudos que comprovem esse impacto em nossa realidade. Há revisões sobre evitabilidade do óbito e seus critérios, sugestão de criação de lista de causas de mortes evitáveis extraídas do contexto do Sistema Único de Saúde (SUS) e efetividade de intervenções para redução da MI,20-22 mas a relação entre a implantação dos CIOI e níveis de mortalidade ou organização dos serviços permanece pouco estudada.

Embora não reflita um diagnóstico recente de implantação dessa estratégia e que modificações tenham ocorrido no Estado de SP desde a finalização da pesquisa, os resultados apontam dificuldades ainda atuais relacionadas à vigilância do óbito e à organização de comitês de investigação, sendo fundamental a superação dessas dificuldades, para que possa contar efetivamente com uma ferramenta a mais na redução da MI

11. Brasil. Ministério da Saúde. Portaria n 399 de 22/02/2006. Divulga o pacto pela saúde 2006 - Consolidação do SUS e aprova as Diretrizes Operacionais do Referido Pacto.

12. São Paulo. Secretaria de Estado da Saúde. Coordenadoria de planejamento em saúde.Centro técnico de saúde da criança. Manual prático dos comitês de mortalidade infantil. Manual técnico. São Paulo; 2000. Disponível em: www.isaude.sp.gov.br

13. Mansano NH, Mazza VA, Soares VMN, Araldi MAR, Cabral VLM. Comitês de prevenção da mortalidade infantil no Paraná, Brasil: implantação e operacionalização. Cad Saúde Pública. 2004; 20: 329-32.

14. Soares VMN, Martins AL. A trajetória e experiência dos comitês de prevenção da mortalidade materna do Paraná Rev Bras Saúde Matern Infant. 2006; 6: 453-60

15. São Paulo. Secretaria de Estado da Saúde. Resolução SS no 81 de 06/09/2006.

16. Hassen MNA, Victora CG, Knauth DR. Pesquisa qualitativa em saúde: uma introdução ao tema. Porto Alegre: Tomo Editorial; 2000.

17. Nogueira-Martins MCF, Bógus CM. Considerações sobre a metodologia qualitativa como recurso para o estudo das ações de humanização em saúde. Saúde Soc. 2004; 13: 4457

18. Donabedian A. The quality of care. How can it be assessed? JAMA. 1988; 260:1743-8.

19. Frias PG, Lira PIC, Vidal SA, Vanderlei LC. Vigilância de óbitos infantis como indicador da efetividade do sistema de saúde - estudo em um município do interior do Nordeste brasileiro. J Pediatr (Rio J). 2002; 78: 509-16 
20. Lansky S, França E, Leal M C. Mortalidade perinatal e evitabilidade: revisão da literatura. Rev Saúde Pública. 2002; 36: 759-72.

21. Malta DC, Duarte EC. Causas de mortes evitáveis por ações efetivas dos serviços de saúde: uma revisão da literatura Ciênc Saúde Coletiva. 2007; 12: 765-76

22. Agencia de Evaluación de Tecnologías Sanitarias (AETS) Instituto de Salud Carlos III - Ministerio de Ciencia e

Recebido em 13 de julho de 2009

Versão final apresentada em 30 de junho de 2010

Aprovado em 12 de agosto de 2010
Innovación. De la Cruz Saugar G, Sarría Santamera A, "Evaluación de intervenciones para la reducción de la mortalidad infantil y materna en países en desarrollo". Madrid: AETS - Instituto de Salud Carlos III, Septiembre de 2008. [Acesso em $10 \mathrm{dez}$ 2009]. Disponível em http://www.isciii.es/htdocs/investigacion/publicaciones_ag encia/AETS 55.pdf. 